\title{
Maximal regularity of discrete and continuous time evolution equations
}

\author{
by
}

Sönke Blunck (Cergy-Pontoise)

\begin{abstract}
We consider the maximal regularity problem for the discrete time evolution equation $u_{n+1}-T u_{n}=f_{n}$ for all $n \in \mathbb{N}_{0}, u_{0}=0$, where $T$ is a bounded operator on a UMD space $X$. We characterize the discrete maximal regularity of $T$ by two types of conditions: firstly by R-boundedness properties of the discrete time semigroup $\left(T^{n}\right)_{n \in \mathbb{N}_{0}}$ and of the resolvent $R(\lambda, T)$, secondly by the maximal regularity of the continuous time evolution equation $u^{\prime}(t)-A u(t)=f(t)$ for all $t>0, u(0)=0$, where $A:=T-I$. By recent results of Weis, this continuous maximal regularity is characterized by R-boundedness properties of the continuous time semigroup $\left(e^{t(T-I)}\right)_{t>0}$ and again of the resolvent $R(\lambda, T)$.

As an important tool we prove an operator-valued Mikhlin theorem for the torus $\mathbb{T}$ providing conditions on a symbol $M \in L_{\infty}(\mathbb{T} ; \mathfrak{L}(X))$ such that the associated Fourier multiplier $T_{M}$ is bounded on $l_{p}(X)$.
\end{abstract}

1. Introduction and main results. The well known problem of maximal $L_{p}$-regularity for continuous time evolution equations is the following. Let $X$ be a Banach space and $\mathcal{T}: \mathbb{R}_{+} \rightarrow \mathfrak{L}(X)$ a bounded analytic semigroup with generator $A$. We consider the evolution equation

$$
u^{\prime}(t)-A u(t)=f(t) \quad \text { for all } t \in \mathbb{R}_{+}, \quad u(0)=0,
$$

where $f: \mathbb{R}_{+} \rightarrow X$ is given and one looks for the solution $u$ on $\mathbb{R}_{+}$which is formally the convolution $u:=\mathcal{T} * f$ on $\mathbb{R}_{+}$. Then $u^{\prime}=\mathcal{T}^{\prime} * f=: \mathcal{R}_{\mathrm{c}} f$ and one says that $A$ has maximal regularity if $\mathcal{R}_{\mathrm{c}} \in \mathcal{L}\left(L_{p}\left(\mathbb{R}_{+} ; X\right)\right)$ for some (and then all $[\mathrm{CL}],[\mathrm{CV}]) p \in(1, \infty)$. Since $\widehat{\mathcal{T}}^{\prime}(\xi)=i \xi R(i \xi, A)-I, \xi \in \mathbb{R}$, the latter is equivalent to the boundedness on $L_{p}(\mathbb{R} ; X)$ of the Fourier multiplier with the operator-valued symbol

$$
\mathbb{R} \ni \xi \mapsto i \xi R(i \xi, A) \in \mathfrak{L}(X) .
$$

This is reflected by the following recent characterization of maximal regularity which is due to Weis [W1].

Theorem A. Let $X$ be a UMD space and let $\left(e^{t A}\right)_{t>0}$ be a bounded analytic semigroup on $X$. Then the following are equivalent:

2000 Mathematics Subject Classification: 39A12, 42A45, 47D06. 
(a) A has maximal regularity.

(b) $\{\lambda R(\lambda, A): \lambda \in i \mathbb{R}, \lambda \neq 0\}$ is R-bounded.

(c) $\left\{e^{t A}, t A e^{t A}: t>0\right\}$ is R-bounded.

We recall that a Banach space $X$ is a UMD space if and only if the classical Hilbert kernel defines a bounded convolution operator on $L_{p}(\mathbb{R} ; X)$ for all $p \in(1, \infty)$.

We use the notion of R-boundedness which was already implicitly used in [Bou] and was introduced in [BG]. A set $\tau \subset \mathfrak{L}(X)$ is called $R$-bounded if there is a constant $C$ such that for all $n \in \mathbb{N}, T_{1}, \ldots, T_{n} \in \tau$ and $x_{1}, \ldots, x_{n} \in$ $X$ we have

$$
\int_{0}^{1}\left\|\sum_{j=1}^{n} r_{j}(t) T_{j}\left(x_{j}\right)\right\| d t \leq C \int_{0}^{1}\left\|\sum_{j=1}^{n} r_{j}(t) x_{j}\right\| d t,
$$

where $\left(r_{j}\right)$ is a sequence of independent symmetric $\{1,-1\}$-valued random variables on $[0,1]$, e.g. the Rademacher functions. By $R(\tau)$ we denote the smallest constant $C$ such that the above condition holds.

Note that in a Hilbert space $X=H$ every bounded set $\tau \subset \mathfrak{L}(H)$ is R-bounded, hence Theorem A generalizes the well known result that, in a Hilbert space, every generator of a bounded holomorphic semigroup has continuous maximal regularity. The latter property is even characteristic of Hilbert spaces, at least in the class of Banach function spaces [KL].

Now we turn to a discrete version of the maximal regularity problem which was formulated and indicated to the author by T. Coulhon.

We replace in our evolution equation (1) the continuous time $t \in \mathbb{R}_{+}$by the discrete time $n \in \mathbb{Z}_{+}$. More precisely, we replace the derivative $u^{\prime}(t)$ by the difference $u_{n+1}-u_{n}$ and the operator $A$ by a "discrete Laplacian" $T-I$. Then $\mathcal{T}: \mathbb{Z}_{+} \rightarrow \mathfrak{L}(X)$ becomes a bounded discrete semigroup $\mathcal{T}(n)=T^{n}$ for a power-bounded operator $T \in \mathfrak{L}(X)$ and the discrete version of (1) reads

$$
u_{n+1}-u_{n}-(T-I) u_{n}=f_{n} \quad \text { for all } n \in \mathbb{Z}_{+}, \quad u_{0}=0 .
$$

Hence we consider the following natural discrete time evolution equation:

$$
u_{n+1}-T u_{n}=f_{n} \quad \text { for all } n \in \mathbb{Z}_{+}, \quad u_{0}=0 .
$$

Again the sequence $f=\left(f_{n}\right)_{n \in \mathbb{Z}_{+}}$is given and the solution $u$ is the convolution $u_{n+1}:=(\mathcal{T} * f)_{n}$ on $\mathbb{Z}_{+}$. If we define the discrete derivative $s^{\prime}$ of a sequence $s=\left(s_{n}\right)$ by $s^{\prime}:=\left(s_{n+1}-s_{n}\right)$, analogously to the continuous time setting, the question arises if $f \in l_{p}\left(\mathbb{Z}_{+} ; X\right)$ implies $u^{\prime} \in l_{p}\left(\mathbb{Z}_{+} ; X\right)$. In other words, we ask if $\mathcal{R}_{\mathrm{d}} f:=\mathcal{T}^{\prime} * f$ or, more explicitly,

$$
\left(\mathcal{R}_{\mathrm{d}} f\right)_{n}:=\sum_{j=0}^{n}(T-I) T^{j} f_{n-j}, \quad n \in \mathbb{Z}_{+},
$$


defines a bounded operator $\mathcal{R}_{\mathrm{d}} \in \mathcal{L}\left(l_{p}\left(\mathbb{Z}_{+} ; X\right)\right)$. This property of $T \in \mathfrak{L}(X)$ will be shown to be independent of $p \in(1, \infty)$ and if it holds we say that $T$ has discrete maximal regularity. Since $\widehat{\mathcal{T}}^{\prime}(z)=z((z-1) R(z, T)-I)$ for all $z \neq 1$ in the torus $\mathbb{T}$, this is equivalent to the boundedness on $l_{p}(X):=$ $l_{p}(\mathbb{Z} ; X)$ of the Fourier multiplier with the operator-valued symbol

$$
\mathbb{T} \ni z \mapsto(z-1) R(z, T) .
$$

In analogy to the continuous time case, we show that a necessary - and in Hilbert spaces $X=H$ sufficient - condition for discrete maximal regularity is that the operator $T$ is analytic in the sense of [C-SC]:

$$
\left\{n(T-I) T^{n}: n \in \mathbb{N}\right\} \text { is bounded. }
$$

This notion is a discrete analogue of the property " $\left\{t A e^{t A}: t>0\right\}$ is bounded", which characterizes the analyticity of a bounded semigroup $\left(e^{t A}\right)_{t \geq 0}$. The following characterization of discrete analyticity is essentially due to O. Nevanlinna; see Theorem 2.3 below. We denote by $\mathbb{D}$ the unit disk in $\mathbb{C}$.

Theorem B. Let $X$ be a Banach space and let $T \in \mathfrak{L}(X)$ be powerbounded, in particular $\sigma(T) \subset \overline{\mathbb{D}}$. Then the following are equivalent:

(i) $\{(\lambda-1) R(\lambda, T):|\lambda|=1, \lambda \neq 1\}$ is bounded.

(ii) $\left\{T^{n}, n(T-I) T^{n}: n \in \mathbb{N}\right\}$ is bounded.

(iii) $\left\{e^{t(T-I)}, t(T-I) e^{t(T-I)}: t>0\right\}$ is bounded and $\sigma(T) \subset \mathbb{D} \cup\{1\}$.

Roughly speaking, our main result below is a combination of an Rboundedness version of Theorem B and an application of Theorem A to the operator $A:=T-I$.

TheOREM 1.1. Let $X$ be a UMD space and let $T \in \mathfrak{L}(X)$ be powerbounded and analytic. Then the following conditions are equivalent:

(a) $T$ has discrete maximal regularity.

(b) $\{(\lambda-1) R(\lambda, T):|\lambda|=1, \lambda \neq 1\}$ is R-bounded.

(c) $\left\{T^{n}, n(T-I) T^{n}: n \in \mathbb{N}\right\}$ is R-bounded.

(d) $A:=T-I$ has maximal regularity.

(e) $\{(\lambda-1) R(\lambda, T): \lambda \in 1+i \mathbb{R}, \lambda \neq 1\}$ is R-bounded.

(f) $\left\{e^{t(T-I)}, t(T-I) e^{t(T-I)}: t>0\right\}$ is R-bounded.

As an application of Theorem 1.1 we obtain the following discrete analogue of a result due to Weis [W2] and (in a slightly weaker version) Lamberton [L] saying that the operator $A$ on $L_{p}$ has maximal regularity if $\left(e^{t A}\right)$ is a subpositive analytic contractive semigroup.

Theorem 1.2. Let $p \in(1, \infty)$ and $T \in \mathfrak{L}\left(L_{p}\right)$ be a subpositive analytic contraction. Then $T$ has discrete maximal regularity. 
Here the subpositivity of a contraction $T$ [resp. of a contractive $C_{0^{-}}$ semigroup $\left(e^{t A}\right)$ ] on $L_{p}$ is defined by the existence of a dominating positive contraction $S$ [resp. of a dominating positive contractive $C_{0}$-semigroup $\left.\left(e^{t B}\right)\right]$, i.e.

$$
|T f| \leq S|f| \quad\left[\text { resp. } \forall t>0:\left|e^{t A} f\right| \leq e^{t B}|f|\right] \quad \text { for all } f \in L_{p} .
$$

Proof of Theorem 1.2. Theorem A yields that $\left(e^{t(T-I)}\right)$ is a subpositive analytic contractive semigroup on $L_{p}$, hence $A:=T-I$ has maximal regularity due to the result of Lamberton and Weis we just mentioned. Thus condition (d) of Theorem 1.1 is satisfied and we deduce the discrete maximal regularity of $T$.

Our Theorem 1.2 shows that e.g. all Markov operators $T$ have discrete maximal regularity on $L_{p}$ for all $p \in(1, \infty)$. This includes random walks on graphs as considered in $[\mathrm{H}-\mathrm{SC}],[\mathrm{CG}],[\mathrm{C}]$ and the references given there.

Further applications of Theorem 1.1 for discrete maximal regularity on $L_{p}$-spaces are given in [B].

The implications $(\mathrm{b}) \Rightarrow(\mathrm{a})$ of Theorem $\mathrm{A}$ and Theorem 1.1 are applications of operator-valued Mikhlin theorems to the Fourier multipliers for the symbols in (2) and (4). The version for multipliers on $\mathbb{R}$ is due to Weis [W1]; here we will prove the following version for multipliers on $\mathbb{T}$ by adapting the proof in [W1].

Theorem 1.3. Let $p \in(1, \infty)$ and $X$ be a UMD space. Let $I:=(-\pi, 0)$ $\cup(0, \pi)$ and $M: I \rightarrow \mathfrak{L}(X)$ be a differentiable function such that the collection

$$
\tau:=\left\{M(t),\left(e^{i t}-1\right)\left(e^{i t}+1\right) M^{\prime}(t): t \in I\right\} \text { is R-bounded. }
$$

Then $T_{M} \in \mathfrak{L}\left(l_{p}(X)\right)$ for the following Fourier multiplier $T_{M}$ :

$\mathcal{F}\left(T_{M} f\right)\left(e^{i t}\right):=M(t) \mathcal{F} f\left(e^{i t}\right), \quad t \in I, \mathcal{F} f \in L_{\infty}(\mathbb{T} ; X)$ of compact support. Moreover, there exists a constant $C_{p, X}$ independent of $M$ such that

$$
\left\|T_{M}\right\|_{\mathfrak{L}\left(l_{p}(X)\right)} \leq C_{p, X} R(\tau) .
$$

The converse implications $(\mathrm{a}) \Rightarrow(\mathrm{b})$ of Theorem A and Theorem 1.1 are seen from the following general criterion for the R-boundedness of Fourier multipliers.

Proposition 1.4. Let $p \in(1, \infty)$ and $G$ be a LCA group with Haar measure $\mu$. Let the dual group $(\widehat{G}, \widehat{\mu})$ be equipped with a translation invariant metric $\widehat{d}$ such that

$$
\begin{aligned}
\sup _{n \in \mathbb{N}} \widehat{\mu}\left(B_{\widehat{G}}\left(e, n^{-1}\right)\right)^{-1}\left\|\mathcal{F}^{-1}\left(\chi_{B_{\widehat{G}}\left(e, n^{-1}\right)}\right)\right\|_{L_{p}(G)} & \\
\times & \times \mathcal{F}^{-1}\left(\chi_{B_{\widehat{G}}\left(e, n^{-1}\right)}\right) \|_{L_{p^{\prime}}(G)}<\infty,
\end{aligned}
$$


where $B_{\widehat{G}}\left(e, n^{-1}\right)$ denotes the ball in $\widehat{G}$ around its identity e of radius $n^{-1}$. Let $X$ be a Banach space and $M \in L_{1, \mathrm{loc}}(\widehat{G} ; \mathfrak{L}(X))$ be such that

$$
\mathcal{F}\left(T_{M} f\right):=M \mathcal{F} f, \quad \mathcal{F} f \in L_{\infty}(\widehat{G} ; X) \text { of compact support, }
$$

defines a bounded operator $T_{M} \in \mathfrak{L}\left(L_{p}(G ; X)\right)$. Then the collection $\{M(\varrho)$ : $\varrho \in \mathcal{L}\}$ is $R$-bounded, where $\mathcal{L}$ denotes the set of Lebesgue points of $M$.

It is easily seen that condition (7) holds e.g. if $G \in\left\{\mathbb{R}^{N}, \mathbb{T}^{N}, \mathbb{Z}^{N}\right\}$ for some $N \in \mathbb{N}$ (see Section 5). Proposition 1.4 is motivated by Proposition 1 of $[\mathrm{CP}]$ where the case $G=\mathbb{R}$ is treated.

2. Generalities on discrete maximal regularity. In this section, $X$ denotes a Banach space and $T \in \mathfrak{L}(X)$ a power-bounded operator. We associate with $T$ the $\mathfrak{L}(X)$-valued kernel

$$
k_{T}: \mathbb{Z} \rightarrow \mathfrak{L}(X), \quad n \mapsto \begin{cases}(T-I) T^{n} & \text { for } n \in \mathbb{N}_{0}, \\ 0 & \text { otherwise }\end{cases}
$$

and the corresponding operator on $\mathbb{Z}_{+}$,

$$
K_{T}: l_{1}\left(\mathbb{Z}_{+} ; X\right) \rightarrow l_{\infty}\left(\mathbb{Z}_{+} ; X\right), \quad f \mapsto\left(\sum_{n=0}^{m} k_{T}(n) f_{m-n}\right)_{m \in \mathbb{Z}_{+}} .
$$

Definition 2.1. (a) $T$ is called analytic if $\left\{n k_{T}(n): n \in \mathbb{N}\right\}$ is bounded.

(b) Let $p \in(1, \infty)$. We say that $T$ has maximal $l_{p}$-regularity if $K_{T} \in$ $\mathcal{L}\left(l_{p}\left(\mathbb{Z}_{+} ; X\right)\right)$.

REMARK 2.2. Let $p \in(1, \infty)$. Then the following are equivalent:

(a) $T$ has maximal $l_{p}$-regularity.

(b) The convolution operator $f \mapsto k_{T} * f$ is bounded on $l_{p}(\mathbb{Z} ; X)$.

Proof. This follows from the translation invariance of convolution operators and the fact that

$$
\forall m \in \mathbb{Z}: \quad\left(k_{T} * f\right)(m)= \begin{cases}\sum_{n=0}^{m} k_{T}(n) f_{m-n}, & m \geq 0, \\ 0, & m<0,\end{cases}
$$

for all $f \in l_{p}(\mathbb{Z} ; X)$ supported in $\mathbb{Z}_{+}$, since their translates are dense in $l_{p}(\mathbb{Z} ; X)$.

The following theorem containing Theorem B relates the analyticity of the operator $T$ to the analyticity of the semigroup $\left(e^{t(T-I)}\right)$, which is (cf. $\left.[\mathrm{P}]\right)$ equivalent to

$$
\left\{(\lambda-1) R(\lambda, T): \lambda \in 1+\Sigma_{\delta}\right\} \text { is bounded for some } \delta>\pi / 2,
$$

where $\Sigma_{\delta}$ denotes the open sector $\{z:|\arg (z)|<\delta\}$.

TheOrem 2.3. Let $T \in \mathfrak{L}(X)$. Then the following are equivalent:

(a) $T$ is power-bounded and analytic. 
(b) $\left(e^{t(T-I)}\right)$ is a bounded analytic semigroup and $\sigma(T) \subset \mathbb{D} \cup\{1\}$.

(c) $\left\{(\lambda-1) R(\lambda, T): \lambda \in \overline{\mathbb{D}}^{c} \cup\left(1+\Sigma_{\delta}\right)\right\}$ is bounded for some $\delta>\pi / 2$.

Here $\mathbb{D}$ is the unit disk in $\mathbb{C}$. Theorem 2.3 is essentially due to O. Nevanlinna [N1], [N2]. Related results can be found in [B], [Ly], [NZ].

Proof. (b) $\Rightarrow(\mathrm{a})$. Follows from [N1, Thm. 4.5.4] since (b) implies (8) by well known semigroup theory $[\mathrm{P}]$.

(a) $\Rightarrow(\mathrm{b})$. Let $\left\|T^{n}\right\| \leq M$ and $\left\|(n+1)(T-I) T^{n}\right\| \leq M$ for all $n \in \mathbb{N}_{0}$. Then (b) is seen from

$$
\begin{gathered}
\left\|e^{t(T-I)}\right\| \leq e^{-t} \sum_{n=0}^{\infty} \frac{t^{n}}{n !}\left\|T^{n}\right\| \leq M, \quad t \geq 0, \\
\left\|(T-I) e^{t(T-I)}\right\| \leq e^{-t} \sum_{n=0}^{\infty} \frac{t^{n}}{n !}\left\|(T-I) T^{n}\right\| \leq M / t, \quad t>0,
\end{gathered}
$$

and the fact that $\left\|(T-I) T^{n}\right\| \geq|z-1| \cdot|z|^{n}$ for all $z \in \sigma(T), n \in \mathbb{N}$, by the spectral mapping theorem (the last argument is taken from $[\mathrm{KT}]$ ).

(a) $\Rightarrow$ (c). If $\left\|T^{n}\right\| \leq M$ for all $n \in \mathbb{N}_{0}$, then

$$
\begin{aligned}
\|R(\lambda, T)\| & =\left\|\sum_{n=0}^{\infty} \lambda^{-n-1} T^{n}\right\| \leq M(|\lambda|-1)^{-1} \\
& \leq M_{R}^{\prime}|\lambda-1|^{-1} \quad \text { for all }|\lambda| \geq R>1 .
\end{aligned}
$$

Since the implication $(\mathrm{a}) \Rightarrow(\mathrm{b})$ is already established, we have (8) as mentioned above, and the fact that $\sigma(T) \subset \mathbb{D} \cup\{1\}$ yields the remaining estimate

$$
\|(\lambda-1) R(\lambda, T)\| \leq M_{R, \delta}^{\prime \prime} \quad \text { for all } R \geq|\lambda|>1 \text { with } \lambda \notin 1+\Sigma_{\delta} .
$$

$(\mathrm{c}) \Rightarrow(\mathrm{b})$. Condition (c) obviously implies $\sigma(T) \subset \mathbb{D} \cup\{1\}$ as well as (8), and as mentioned above, the latter is equivalent to the fact that $\left(e^{t(T-I}\right)$ is a bounded analytic semigroup.

The continuous time analogue of the following observation is the fact that every closed densely defined operator in $X$ having maximal $L_{p}$-regularity generates a bounded analytic semigroup.

Proposition 2.4. Let $p \in(1, \infty)$. If $T \in \mathfrak{L}(X)$ is power-bounded and has maximal $l_{p}$-regularity then $T$ is analytic.

Proof. Let $\left\|T^{n}\right\| \leq M$ for all $n \in \mathbb{N}_{0}$. Similarly to the continuous time argument [CL], we consider for all $b \in \mathbb{N}, x \in X$ the sequence $f=f_{b, x} \in$ $l_{p}\left(\mathbb{Z}_{+} ; X\right)$ defined by

$$
f_{j}:= \begin{cases}T^{j} x & \text { for } j=1, \ldots, b \\ 0 & \text { otherwise }\end{cases}
$$


Then for all $n \in \mathbb{N}$ we have

$$
\left(K_{T} f\right)_{n}=\sum_{j=1}^{n} k_{T}(n-j) f_{j}=\sum_{j=1}^{n}(T-I) T^{n-j} f_{j}=(n \wedge b)(T-I) T^{n} x .
$$

The fact that $\|f\|_{p} \leq M b^{1 / p}\|x\|$ combines with

$$
\begin{aligned}
\left\|K_{T} f\right\|_{p} & \geq\left(\sum_{n=1}^{b}\left\|\left(K_{T} f\right)_{n}\right\|^{p}\right)^{1 / p}=\left(\sum_{n=1}^{b} n^{p}\left\|(T-I) T^{n} x\right\|^{p}\right)^{1 / p} \\
& \geq M^{-1}\left(\sum_{n=1}^{b} n^{p}\right)^{1 / p}\left\|(T-I) T^{b} x\right\| \geq(2 M)^{-1} b^{1+1 / p}\left\|(T-I) T^{b} x\right\|
\end{aligned}
$$

to give the estimate $\left\|(T-I) T^{b} x\right\| \leq 2 M^{2}\left\|K_{T}\right\|_{p, p} b^{-1}\|x\|$.

With the aid of the following well known vector-valued BenedekCalderón-Panzone Theorem [BCP], we obtain the same $p$-independence of maximal regularity as in the continuous time version.

TheOREM 2.5. Let $k \in l_{\infty}(\mathfrak{L}(X))$ and $q \in[1, \infty]$. Let $S \in \mathcal{L}\left(l_{q}(X)\right)$ be such that

$$
(S f)_{n}=\sum_{m \in \mathbb{Z}} k(n-m) f_{m} \quad \text { for all } n \in \mathbb{Z}
$$

and for all $f \in l_{1}(X) \cap l_{q}(X)$. If the Hörmander condition

$$
\sup _{m \in \mathbb{Z}} \sum_{|n|>2|m|}\|k(n-m)-k(n)\|<\infty
$$

is satisfied then $S \in \mathcal{L}\left(l_{p}(X)\right)$ for all $p \in(1, \infty)$.

Corollary 2.6. Let $p, q \in(1, \infty)$. If $T$ has maximal $l_{q}$-regularity then $T$ has maximal $l_{p}$-regularity.

Proof. Let $T$ have maximal $l_{q}$-regularity. Then $T$ is analytic by Proposition 2.4, which implies easily

$$
\left\|(T-I)^{2} T^{n}\right\| \leq C / n^{2} \text { for all } n \in \mathbb{N} .
$$

Now the proof of the Hörmander condition (9) is a simple modification of the corresponding proof for the continuous time situation as given e.g. in [D, p. 32].

From now on we will say that $T$ has discrete maximal regularity if $T$ has maximal $l_{p}$-regularity for some $p \in(1, \infty)$.

Proposition 2.7. Let $H$ be a Hilbert space and $T \in \mathcal{L}(H)$ be powerbounded and analytic. Then $T$ has discrete maximal regularity.

Proof. By the $p$-independence just established, it suffices to show that $T$ has maximal $l_{2}$-regularity. But due to Remark 2.2 and the Hilbert space 
situation, this is equivalent to

$$
\mathcal{F}\left(k_{T}\right) \in L_{\infty}(\mathbb{T} ; \mathcal{L}(H)) .
$$

Hence we have to show that

$$
g_{T}: \mathbb{D} \rightarrow \mathcal{L}(H), \quad z \mapsto \sum_{n=0}^{\infty} z^{n} k_{T}(n),
$$

has a bounded extension to $\overline{\mathbb{D}}$. But the latter follows from Theorem 2.3 and

$$
g_{T}(z)=(T-I) \sum_{n=0}^{\infty}(z T)^{n}=z^{-1}\left(\left(z^{-1}-1\right) R\left(z^{-1}, T\right)-I\right) .
$$

3. R-boundedness. The notion of R-boundedness was already implicitly used in [Bou] and was introduced in [BG]. It is fundamental for our purposes since it allows us to generalize many classical results on (scalarvalued) Fourier multipliers to the operator-valued setting on UMD spaces; see e.g. [Bou], [BG], [W1], [SW].

In this section we collect some examples and some operations (e.g. closures, products, sums, means) on R-bounded sets which, roughly speaking, modify their R-bound in the same way as their norm-bound.

Let $X$ be a Banach space and $\left(r_{j}\right)_{j \in \mathbb{N}}$ be a sequence of independent symmetric $\{1,-1\}$-valued random variables on $[0,1]$, e.g. the Rademacher functions.

Definition 3.1. A set $\tau \subset \mathfrak{L}(X)$ is called $R$-bounded if

$$
\int_{0}^{1}\left\|\sum_{j=1}^{n} r_{j}(t) T_{j}\left(x_{j}\right)\right\|^{p} d t \leq C_{p}^{p} \int_{0}^{1}\left\|\sum_{j=1}^{n} r_{j}(t) x_{j}\right\|^{p} d t
$$

for some $p \in[1, \infty), C_{p}>0$ and for all $n \in \mathbb{N}, T_{1}, \ldots, T_{n} \in \tau$ and $x_{1}, \ldots, x_{n} \in X$. Recall that, by Kahane's inequality [LT], this property is independent of $p \in[1, \infty)$. The smallest constant $C_{p}$ for which (10) holds is denoted by $R_{p}(\tau)$, and furthermore we set $R(\tau):=R_{1}(\tau)$.

For the following basic examples and facts we refer to [W1, §2] and [SW, $\S 2]$ and the references given there.

EXAMPLE 3.2. (a) If $H$ is a Hilbert space then $\tau \subset \mathfrak{L}(H)$ is R-bounded if and only if $\tau$ is bounded.

(b) If $\Lambda \subset \mathbb{C}$ is bounded by $M \geq 0$ then $R\left(\left\{\lambda I_{X}: \lambda \in \Lambda\right\}\right) \leq 2 M$.

REMARK 3.3. Let $\tau, \sigma \subset \mathfrak{L}(X)$.

(a) If $\tau$ is R-bounded then the closure of $\tau$ in the strong operator topology is also R-bounded.

(b) Let $\tau$ be $R$-bounded with $R$-bound $M$. Then the closure of the complex absolute convex hull of $\tau$ is also $R$-bounded with $R$-bound at most $2 M$. 
(c) If $\sigma$ and $\tau$ are $R$-bounded then $\sigma \cup \tau$ and $\sigma \tau$ are also $R$-bounded; more precisely:

$$
R(\sigma \cup \tau) \leq R(\sigma)+R(\tau), \quad R(\{S T: S \in \sigma, T \in \tau\}) \leq R(\sigma) R(\tau) .
$$

(d) Let $G \subset \mathbb{C}$ be a simply connected Jordan region such that $\mathbb{C} \backslash G$ has interior points. Let $F \in L_{\infty}(\bar{G} ; \mathfrak{L}(X))$ be analytic in $G$ and $F(\partial G)$ be $R$-bounded. Then $F(\bar{G})$ is also R-bounded.

(e) Let $\Omega$ be a measure space and $p \in[1, \infty)$. For all $T \in \mathfrak{L}(X)$ we define the operator $\widetilde{T} \in \mathfrak{L}\left(L_{p}(\Omega ; X)\right)$ by $(\widetilde{T} f)(\omega):=T(f(\omega)), f \in L_{p}(\Omega ; X)$, $\omega \in \Omega$. Then there exists a constant $C_{p}$ independent of $X$ and $\tau \subset \mathfrak{L}(X)$ such that

$$
R(\{\widetilde{T}: T \in \tau\}) \leq C_{p} R(\tau) .
$$

(f) Let $X=L_{p}(\Omega ; E)$ for some measure space $\Omega$, Banach space $E$ and $p \in[1, \infty)$. If $\tau \subset \mathfrak{L}(X)$ is $R$-bounded then

$$
\left\{\phi T \psi:\|\phi\|_{\infty},\|\psi\|_{\infty} \leq 1, T \in \tau\right\} \text { is R-bounded. }
$$

3.1. R-boundedness and power series

Lemma 3.4. Let $\tau \subset \mathfrak{L}(X)$ be $R$-bounded and $C>0, q \in[0,1)$. Then for $\mathcal{A}:=\left\{a \in l_{\infty}: \forall n \in \mathbb{N}:\left|a_{n}\right| \leq C(q / R(\tau))^{n}\right\}$ the set $\mathcal{M}:=\left\{\sum_{n=1}^{\infty} a_{n} T^{n}:\right.$ $a \in \mathcal{A}, T \in \tau\}$ is R-bounded.

Proof. By Remark 3.3(a) it suffices to show

$$
\int_{0}^{1}\left\|\sum_{j=1}^{m} r_{j}(t) \sum_{n=1}^{N} a_{n}^{(j)} T_{j}^{n} x_{j}\right\| d t \leq \frac{2 C q}{1-q} \int_{0}^{1}\left\|\sum_{j=1}^{m} r_{j}(t) x_{j}\right\| d t
$$

for all $m, N \in \mathbb{N}, a^{(1)}, \ldots, a^{(m)} \in \mathcal{A}, T_{1}, \ldots, T_{m} \in \tau$ and $x_{1}, \ldots, x_{m} \in X$. For $\sigma_{n}:=\left\{a_{n} I_{X}: a \in \mathcal{A}\right\}$ we have, by $3.2(\mathrm{~b})$,

$$
R\left(\sigma_{n}\right) \leq 2 \sup _{a \in \mathcal{A}}\left|a_{n}\right| \leq 2 C(q / R(\tau))^{n},
$$

hence we can estimate as follows:

$$
\begin{aligned}
\int_{0}^{1}\left\|\sum_{j=1}^{m} r_{j}(t) \sum_{n=1}^{N} a_{n}^{(j)} T_{j}^{n} x_{j}\right\| d t & \leq \sum_{n=1}^{N} R(\tau)^{n} \int_{0}^{1}\left\|\sum_{j=1}^{m} r_{j}(t) a_{n}^{(j)} x_{j}\right\| d t \\
& \leq \sum_{n=1}^{N} R(\tau)^{n} R\left(\sigma_{n}\right) \int_{0}^{1}\left\|\sum_{j=1}^{m} r_{j}(t) x_{j}\right\| d t \\
& \leq \frac{2 C q}{1-q} \int_{0}^{1}\left\|\sum_{j=1}^{m} r_{j}(t) x_{j}\right\| d t .
\end{aligned}
$$

In the first step we used Remark 3.3(c) in the form

$$
R\left(\left\{T^{n}: T \in \tau\right\}\right) \leq R(\tau)^{n} \quad \text { for all } n \in \mathbb{N} .
$$


While part (b) of the following corollary is a trivial application of [W1, Prop. 2.6], its part (a) is implicitly shown in the proof of [W1, Thm. 4.2].

Corollary 3.5. Let $A$ be a closed and densely defined operator in $X$.

(a) If $\left\{\lambda R(\lambda, A): \lambda \in \Sigma_{\pi / 2}\right\}$ is R-bounded then there exists $\delta>\pi / 2$ such that $\left\{\lambda R(\lambda, A): \lambda \in \Sigma_{\delta}\right\}$ is R-bounded.

(b) If $G \subset \varrho(A)$ is compact then $\{R(\lambda, A): \lambda \in G\}$ is R-bounded.

Proof. (a) By well known semigroup theory [P] there exists $\delta^{\prime}>\pi / 2$ such that

$$
\left\{\lambda R(\lambda, A): \lambda \in \Sigma_{\delta^{\prime}}\right\} \text { is bounded. }
$$

Hence $\tau:=\left\{\lambda R(\lambda, A): \lambda \in \bar{\Sigma}_{\pi / 2}, \lambda \neq 0\right\}$ is R-bounded by Remark 3.3(a). Now we choose $q \in(0,1)$ arbitrary, $C:=\sqrt{1+(R(\tau) / q)^{2}}$ and $\mathcal{A}, \mathcal{M}$ as in Lemma 3.4. Since $\tau \subset \mathcal{M}$ it suffices to show

$$
|s| /|t| \leq q / R(\tau) \Rightarrow(s+i t) R(s+i t, A) \in \mathcal{M}
$$

But this follows directly from

$$
\begin{aligned}
(s+i t) R(s+i t) & =(s+i t) \sum_{n=0}^{\infty} R(i t, A)^{n+1}(-s)^{n} \\
& =\sum_{n=1}^{\infty} \frac{(s+i t)(-s)^{n-1}}{(i t)^{n}}(i t R(i t, A))^{n}
\end{aligned}
$$

and the elementary implication

$$
|s| /|t| \leq q / R(\tau) \Rightarrow\left(\frac{(s+i t)(-s)^{n-1}}{(i t)^{n}}\right)_{n \in \mathbb{N}} \in \mathcal{A} .
$$

(b) follows directly from [W1, Prop. 2.6].

3.2. $R$-boundedness and functional calculus. Let $X$ be a Banach space and $T \in \mathfrak{L}(X)$. Let $\mathcal{E}$ be the set of all entire $\mathbb{C}$-valued functions. For all $f \in \mathcal{E}$ we define

$$
f(T):=\int_{\Gamma_{f}} f(\lambda) R(\lambda, T) d \lambda
$$

where $\Gamma_{f}$ is an arbitrary path in $\varrho(T)$ around $\sigma(T)$. Note that this definition is independent of the chosen path $\Gamma_{f}$.

Lemma 3.6. Let $\mathcal{M} \subset \varrho(T)$ and $g: \mathcal{M} \rightarrow \mathbb{C} \backslash\{0\}$ be continuous such that $\{g(\lambda) R(\lambda, T): \lambda \in \mathcal{M}\}$ is $R$-bounded. Let $\mathcal{F} \subset \mathcal{E}$ be such that $\Gamma_{f} \subset \mathcal{M}$ for all $f \in \mathcal{F}$ and

$$
\sup _{f \in \mathcal{F}} \int_{\Gamma_{f}}\left|f(\lambda) g(\lambda)^{-1}\right||d \lambda|<\infty .
$$

Then the set $\{f(T): f \in \mathcal{F}\}$ is $R$-bounded. 
Proof. If we write $\tau:=\{g(\lambda) R(\lambda, T): \lambda \in \mathcal{M}\}$ and

$$
C_{f}:=\int_{\Gamma_{f}}\left|f(\lambda) g(\lambda)^{-1}\right||d \lambda|, \quad f \in \mathcal{F},
$$

then obviously the operators $C_{f}^{-1} f(T)$ belong to the closure of the complex absolute convex hull of $\tau$, which is R-bounded by Remark 3.3(b). Hence the assertion follows from $3.2(\mathrm{~b})$ :

$$
R(\{f(T): f \in \mathcal{F}\}) \leq 2\left(\sup _{f \in \mathcal{F}} C_{f}\right) R\left(\left\{C_{f}^{-1} f(T): f \in \mathcal{F}\right\}\right) .
$$

The following proposition is the implication $(\mathrm{b}) \Rightarrow(\mathrm{c})$ of Theorem 1.1.

Proposition 3.7. Let $T \in \mathfrak{L}(X)$ be power-bounded and analytic. Then $\left\{T^{n}, n(T-I) T^{n}: n \in \mathbb{N}\right\}$ is $R$-bounded if $\{(\lambda-1) R(\lambda, T):|\lambda|=1, \lambda \neq 1\}$ is R-bounded.

Proof. By Nevanlinna's Theorem 2.3 and the "maximum principle" Remark $3.3(\mathrm{~d})$, the hypotheses imply that $\left\{(\lambda-1) R(\lambda, T): \lambda \in \mathbb{D}^{\mathrm{c}}, \lambda \neq 1\right\}$ is R-bounded. Hence, due to the "sector extension" Corollary 3.5(a), we find some $\delta>0$ such that $\left\{(\lambda-1) R(\lambda, T): \lambda \in \mathbb{D}^{\mathrm{C}} \cup\left(1+\Sigma_{\delta}\right), \lambda \neq 1\right\}$ is R-bounded. Moreover, there exists $\varepsilon>0$ such that $K:=\{\lambda: \operatorname{dist}(\lambda, \partial \mathbb{D} \backslash$ $\left.\left.\left(1+\bar{\Sigma}_{\delta}\right)\right) \leq \varepsilon\right\} \subset \varrho(T)$. Since $K$ is compact, we see from Corollary $3.5(\mathrm{~b})$ for $\mathcal{M}:=\overline{\mathbb{D}}^{c} \cup\left(1+\Sigma_{\delta}\right) \cup K$ that $\{(\lambda-1) R(\lambda, T): \lambda \in \mathcal{M}\}$ is R-bounded.

Now, roughly speaking, Nevanlinna's functional calculus argument in [N1, p. 102] establishing

$$
\{(\lambda-1) R(\lambda, S): \lambda \in \mathcal{M}\} \text { bd } \Rightarrow\left\{S^{n}, n(S-I) S^{n}: n \in \mathbb{N}\right\} \text { bd }
$$

for any $S \in \mathfrak{L}(X)$ and our Lemma 3.6 show that (11) remains true if "bd" = bounded is replaced by "R-bounded". For the convenience of the reader we give a detailed proof. One checks that for sufficiently small $t_{0}, c_{0}>0$ the map

$$
\left[0, t_{0}\right] \rightarrow \mathbb{R}_{+}, \quad t \mapsto\left|1+s+t e^{i(\delta+\pi) / 2}\right| \mid 1-c_{0} t^{-1},
$$

is decreasing for all $s \in[0,1]$. Hence we have

$$
\left|1+s+t e^{i(\delta+\pi) / 2}\right| \leq(1+s)\left(1-c_{0} t\right) \quad \text { for all } t \in\left[0, t_{0}\right], s \in[0,1] .
$$

Now choose $t_{\infty} \in\left[0, t_{0} / 2\right]$ such that $z_{0}:=1+t_{\infty} e^{i(\delta+\pi) / 2}$ satisfies $r:=\left|z_{0}\right|$ $<1$ and

$$
\mathcal{A}:=\left\{z:|z|=r,|\arg (z)| \geq \arg \left(z_{0}\right)\right\} \subset \mathcal{M} .
$$

Then we find $k_{0}>0$ such that for all $k \geq k_{0}$ we have

$$
\exists t_{k} \in\left[0, t_{0}\right]: \quad 1+k^{-1}+t_{k} e^{i(\delta+\pi) / 2} \in \mathcal{A} .
$$


For all such $k \geq k_{0}$ we construct a path $\Gamma_{k}$ as follows:

$$
\begin{aligned}
\Gamma_{k} & =\Gamma_{k, 1} \cup \Gamma_{k, 2} \cup \Gamma_{k, 3}, \\
\Gamma_{k, 1} & :=1+k^{-1}+\left[0, t_{k}\right] e^{i(\delta+\pi) / 2}, \\
\Gamma_{k, 2} & \subset \mathcal{A}, \\
\Gamma_{k, 3} & :=1+k^{-1}+\left[0, t_{k}\right] e^{-i(\delta+\pi) / 2} .
\end{aligned}
$$

Observe that $\Gamma_{k} \subset \mathcal{M}$. Hence we will apply Lemma 3.6 to $g(\lambda):=\lambda-1$, $\mathcal{F}:=\left\{f_{n}, \widetilde{f}_{n}: n \in \mathbb{N}\right\}$ and $\Gamma_{f_{n}}:=\Gamma_{n+k_{0}}=: \Gamma_{\widetilde{f}_{n}}$, where $f_{n}(\lambda):=\lambda^{n}$ and $\widetilde{f}_{n}(\lambda):=n(\lambda-1) \lambda^{n}$. This yields the R-boundedness of $\left\{T^{n}, n(T-I) T^{n}\right.$ : $n \in \mathbb{N}\}$ once we show

$$
\sup _{n \in \mathbb{N}} \int_{\Gamma_{n+k_{0}}}|\lambda|^{n}\left(n+|\lambda-1|^{-1}\right)|d \lambda|<\infty .
$$

The integrals over $\Gamma_{n+k_{0}, 1}$ are estimated as follows by using (12):

$$
\begin{aligned}
\int_{\Gamma_{n+k_{0}, 1}}|\lambda|^{n}|d \lambda| & \leq \int_{0}^{t_{0}}\left(1+\left(n+k_{0}\right)^{-1}\right)^{n}\left(1-c_{0} t\right)^{n} d t \\
& \leq e \int_{0}^{t_{0}}\left(1-c_{0} t\right)^{n} d t \leq C_{1} / n \quad \text { for all } n \in \mathbb{N} \\
\int_{\Gamma_{n+k_{0}, 1}|\lambda|^{n}|\lambda-1|^{-1}|d \lambda|} & \leq \int_{0}^{t_{0}} \frac{\left(1+\left(n+k_{0}\right)^{-1}\right)^{n}\left(1-c_{0} t\right)^{n}}{\left|\left(n+k_{0}\right)^{-1}+t e^{i(\delta+\pi) / 2}\right|} d t \\
& \leq e \int_{0}^{\infty} \frac{e^{-c_{0} \tau /\left(1+k_{0}\right)}}{\left|1+\tau e^{i(\delta+\pi) / 2}\right|} d \tau \quad \text { for all } n \in \mathbb{N}
\end{aligned}
$$

For the integrals over $\Gamma_{n+k_{0}, 2}$ we even have exponential decay in $n$ :

$$
\begin{gathered}
\int_{\Gamma_{n+k_{0}, 2}}|\lambda|^{n}|d \lambda| \leq \int_{|\lambda|=r} r^{n}|d \lambda|=C_{2} r^{n} \quad \text { for all } n \in \mathbb{N}, \\
\int_{\Gamma_{n+k_{0}, 2}}|\lambda|^{n}|\lambda-1|^{-1}|d \lambda| \leq \int_{|\lambda|=r} r^{n}(1-r)^{-1}|d \lambda|=C_{3} r^{n} \quad \text { for all } n \in \mathbb{N} .
\end{gathered}
$$

Since the integrals over $\Gamma_{n+k_{0}, 3}$ are symmetric to $\Gamma_{n+k_{0}, 1}$, the proof is complete.

4. Fourier multipliers on the torus. The aim of this section is the proof of the Mikhlin Theorem 1.3 for operator-valued Fourier multipliers on the torus $\mathbb{T}$. It is an adaptation of the corresponding proof for multipliers on $\mathbb{R}$ as given by Weis [W1]. 


\subsection{Dyadic decomposition}

Definition 4.1. (a) We first decompose $(0, \pi)$ "dyadically" into the following family $\left(I_{j}\right)_{j \in \mathbb{Z}}$ of intervals:

$$
I_{j}:= \begin{cases}{\left[\pi-2^{-(j+1)} \pi, \pi-2^{-(j+2)} \pi\right),} & j \geq 0, \\ {\left[2^{j-1} \pi, 2^{j} \pi\right),} & j<0 .\end{cases}
$$

(b) Now we denote by $a_{j}, b_{j}, \Delta_{j}$ the endpoints and the corresponding $\operatorname{arcs}$ of $I_{j}$ :

$$
I_{j}=\left[a_{j}, b_{j}\right) \quad \text { and } \quad \Delta_{j}:=\left\{e^{i t}: t \in-I_{j} \cup I_{j}\right\} .
$$

The following lemma will be useful later. Its proof is elementary and therefore omitted.

LEMmA 4.2. There exists a constant $D_{1}$ such that $\left|1-e^{i\left[a_{j}+r\left(b_{j}-a_{j}\right)\right]}\right|^{-1}\left|1+e^{i\left[a_{j}+r\left(b_{j}-a_{j}\right)\right]}\right|^{-1} \leq D_{1} 2^{|j|} \quad$ for all $j \in \mathbb{Z}, r \in[0,1]$.

4.2. A Marcinkiewicz-type Multiplier Theorem

TheOREM 4.3. Let $X$ be a UMD space and $p \in(1, \infty)$. Then, for all $M: \mathbb{T} \rightarrow \mathfrak{L}(X)$ of the form $M=\sum_{j \in \mathbb{Z}} \chi_{\Delta_{j}} m M_{j}$, where $m: \mathbb{T} \rightarrow \mathbb{C}$ has uniformly bounded variations over the $\left(\Delta_{j}\right)_{j \in \mathbb{Z}}$ and $\left\{M_{j}: j \in \mathbb{Z}\right\} \subset \mathfrak{L}(X)$ is $R$-bounded, we have

$$
\left\|T_{M}\right\|_{\mathfrak{L}\left(l_{p}(X)\right)} \leq C_{p, X} R\left(\left\{M_{j}: j \in \mathbb{Z}\right\}\right) \sup _{j \in \mathbb{Z}} \operatorname{Var}_{\Delta_{j}} m .
$$

Here we write $\operatorname{Var}_{\Delta_{j}} m:=\max \left(\operatorname{var}_{\Delta_{j}} m,\|m\|_{L_{\infty}\left(\Delta_{j}\right)}\right)$, where $\operatorname{var}_{\Delta_{j}} m$ is the usual variation of $m$ over $\Delta_{j}$.

Proof. From the so-called Littlewood-Paley property of the dyadic decomposition $\left\{\left(-2^{j+1},-2^{j}\right] \cup\left[2^{j}, 2^{j+1}\right): j \in \mathbb{Z}\right\}$ of $\mathbb{R}$ (see [W1, Thm. 3.1]), we obtain by a standard transference argument [BG, Thm. 3.6(iii)] the Littlewood-Paley property of our dyadic decomposition of $\mathbb{T}$ :

$$
C^{-1}\|f\|_{l_{p}(X)} \leq \int_{0}^{1}\left\|\sum_{j \in \mathbb{Z}} r_{j}(t) S_{j}(f)\right\|_{l_{p}(X)} d t \leq C\|f\|_{l_{p}(X)} .
$$

Here the $S_{j}$ are the Fourier multipliers $\mathcal{F}\left(S_{j} f\right):=\chi_{\Delta_{j}} \mathcal{F} f$, which are often called "partial sum operators". In combination with the extension result Remark 3.3(e) we can estimate as follows, using the symbol $\preceq$ to express domination up to constants depending only on $p$ and $X$ :

$$
\begin{aligned}
\left\|T_{M} f\right\|_{l_{p}(X)} & \preceq \int_{0}^{1}\left\|\sum_{j \in \mathbb{Z}} r_{j}(t) S_{j}\left(T_{M} f\right)\right\|_{l_{p}(X)} d t \\
& =\int_{0}^{1}\left\|\sum_{j \in \mathbb{Z}} r_{j}(t) \widetilde{M}_{j}\left(S_{j}\left(T_{m} f\right)\right)\right\|_{l_{p}(X)} d t
\end{aligned}
$$




$$
\begin{aligned}
& \preceq R\left(\left\{M_{j}: j \in \mathbb{Z}\right\}\right) \int_{0}^{1}\left\|\sum_{j \in \mathbb{Z}} r_{j}(t) S_{j}\left(T_{m} f\right)\right\|_{l_{p}(X)} d t \\
& \preceq R\left(\left\{M_{j}: j \in \mathbb{Z}\right\}\right)\left\|T_{m} f\right\|_{l_{p}(X)} \\
& \leq R\left(\left\{M_{j}: j \in \mathbb{Z}\right\}\right)\left\|T_{m}\right\|_{\mathfrak{L}\left(l_{p}(X)\right)}\|f\|_{l_{p}(X)} \\
& \preceq R\left(\left\{M_{j}: j \in \mathbb{Z}\right\}\right)\left\|T_{m}\right\|_{\mathfrak{L}\left(l_{p}\right)}\|f\|_{l_{p}(X)} \\
& \preceq R\left(\left\{M_{j}: j \in \mathbb{Z}\right\}\right) \sup _{j} \operatorname{Var}_{\Delta_{j}} m\|f\|_{l_{p}(X)} .
\end{aligned}
$$

For the last two steps we used [BG, Thm. 4.5] and the Marcinkiewicz Multiplier Theorem for $\mathbb{T}$ in its classical, i.e. scalar-valued version [EG].

4.3. Proof of the Mikhlin Multiplier Theorem 1.3. We approximate the given symbol $M$ by linear combinations $M_{k}, k \in \mathbb{N}$, of symbols of the type considered in our Marcinkiewicz-type Theorem 4.3: For all $t \in(-\pi, 0) \cup(0, \pi)$ we define

$$
M_{k}\left(e^{i \theta}\right):=\sum_{j \in \mathbb{Z}, \sigma= \pm 1} \chi_{\sigma I_{j}}(t)\left(M\left(\sigma a_{j}\right)+\sigma \sum_{l=1}^{2^{k}} \chi_{\sigma\left[a_{j}, b_{j, k, l}\right)}(t) \delta_{j, k} M^{\prime}\left(\sigma b_{j, k, l}\right)\right) .
$$

Here we choose $b_{j, k, l}:=a_{j}+(l-1) \delta_{j, k}$ and

$$
\delta_{j, k}:=2^{-k}\left(b_{j}-a_{j}\right)= \begin{cases}2^{-k-|j|-2} \pi, & j \geq 0 \\ 2^{-k-|j|-1} \pi, & j<0\end{cases}
$$

By condition (6) and Lemma 4.2, the $M_{k}$ are uniformly bounded on $\mathbb{T} \backslash\{ \pm 1\}$. Moreover, for all $j \in \mathbb{Z}$ and for all $t \in \sigma I_{j}=\sigma\left[a_{j}, b_{j}\right)$ we have

$$
\begin{aligned}
M_{k}\left(e^{i t}\right) & =M\left(\sigma a_{j}\right)+\sigma \sum_{l=1}^{2^{k}} \chi_{\sigma\left[a_{j}, b_{j, k, l}\right)}(t) \delta_{j, k} M^{\prime}\left(\sigma b_{j, k, l}\right) \\
& \stackrel{k}{\rightarrow} M\left(\sigma a_{j}\right)+\int_{\sigma a_{j}}^{t} M^{\prime}(s) d s=M(t) .
\end{aligned}
$$

Hence, in order to show $T_{M} \in \mathfrak{L}\left(l_{p}(X)\right)$ and the desired estimate in the norm of $\mathfrak{L}\left(l_{p}(X)\right)$, it suffices to show

$$
\left\|T_{M_{k}}\right\|_{\mathfrak{L}\left(l_{p}(X)\right)} \leq C_{p, X} R(\tau) \quad \text { for all } k \in \mathbb{N} .
$$

For this purpose, we decompose the symbols $M_{k}$ as

$$
M_{k}=\sum_{\sigma= \pm 1}\left(M_{k, 0, \sigma}+2^{-k} \sigma \sum_{l=1}^{2^{k}} M_{k, l, \sigma}\right)
$$


where the $M_{k, l, \sigma}$ are symbols of the type considered in our Marcinkiewicztype Multiplier Theorem 4.3:

$$
M_{k, l, \sigma}:=\sum_{j \in \mathbb{Z}} \chi_{\Delta_{j}} m^{(k, l, \sigma)} M_{j}^{(k, l, \sigma)}, \quad k \in \mathbb{N}, l=0, \ldots, 2^{k}, \sigma= \pm 1,
$$

where the $m^{(k, l, \sigma)}$ and the $M_{j}^{(k, l, \sigma)}$ are given by

$$
\begin{aligned}
m^{(k, l, \sigma)}\left(e^{i \theta}\right) & := \begin{cases}1, & l=0, \\
\sum_{r \in \mathbb{Z}} \chi_{\sigma\left[a_{r}, b_{r, k, l}\right)}(t), & l=1, \ldots, 2^{k},\end{cases} \\
M_{j}^{(k, l, \sigma)} & := \begin{cases}M\left(\sigma a_{j}\right), & l=0, \\
2^{k} \delta_{j, k} M^{\prime}\left(\sigma b_{j, k, l}\right), & l=1, \ldots, 2^{k} .\end{cases}
\end{aligned}
$$

Our aim (14) is clear once we show

$$
R\left(\left\{M_{j}^{(k, l, \sigma)}: j \in \mathbb{Z}\right\}\right) \leq C R(\tau)
$$

for all $k \in \mathbb{N}, l=0, \ldots, 2^{k}, \sigma= \pm 1$.

Indeed, the fact that $\operatorname{Var}_{\Delta_{j}} m^{(k, l, \sigma)} \leq 1$ for all $j, k, l, \sigma$ under consideration, the line (16) and Theorem 4.3 combine to

$$
\left\|T_{M_{k, l, \sigma}}\right\|_{\mathfrak{L}\left(l_{p}(X)\right)} \leq C_{p, X}^{\prime} R(\tau) \quad \text { for all } k \in \mathbb{N}, l=0, \ldots, 2^{k}, \sigma= \pm 1,
$$

so that $\left\|T_{M_{k}}\right\| \leq 4 C_{p, X}^{\prime} R(\tau)$ by the decomposition (15). For the case $l=0$, the estimate (16) holds trivially for $C=1$, for the other cases it is seen from

$$
\begin{aligned}
& \sup _{k \in \mathbb{N}, l \in\left\{1, \ldots, 2^{k}\right\}, \sigma= \pm 1} R\left(\left\{M_{j}^{(k, l, \sigma)}: j \in \mathbb{Z}\right\}\right) \\
& =\sup _{k \in \mathbb{N}, l \in\left\{1, \ldots, 2^{k}\right\}, \sigma= \pm 1} R\left(\left\{\left(b_{j}-a_{j}\right) M^{\prime}\left(\sigma a_{j}+\sigma 2^{-k}(l-1)\left(b_{j}-a_{j}\right)\right): j \in \mathbb{Z}\right\}\right) \\
& \leq \sup _{r \in[0,1], \sigma= \pm 1} R\left(\left\{\left(b_{j}-a_{j}\right) M^{\prime}\left(\sigma a_{j}+\sigma r\left(b_{j}-a_{j}\right)\right): j \in \mathbb{Z}\right\}\right) \\
& \leq \pi D_{1} \sup _{r \in[0,1], \sigma= \pm 1} R\left(\left\{\left(1-e^{i \sigma\left[a_{j}+r\left(b_{j}-a_{j}\right)\right]}\right)\left(1+e^{i \sigma\left[a_{j}+r\left(b_{j}-a_{j}\right)\right]}\right)\right.\right. \\
& \left.\left.\quad \times M^{\prime}\left(\sigma a_{j}+\sigma r\left(b_{j}-a_{j}\right)\right): j \in \mathbb{Z}\right\}\right) \\
& \leq \pi D_{1} R(\tau) .
\end{aligned}
$$

Here we used (13), Lemma 4.2 and 3.2(a) for the third step.

5. Necessity of R-boundedness. Recall that $G$ is a LCA group with Haar measure $\mu$ and character group $(\widehat{G}, \widehat{\mu})$, equipped with a translation invariant metric $\widehat{d}$. By $e$ and $B(\varrho, r)$ we denote the identity and balls in $\widehat{G}$.

Proof of Proposition 1.4. Setting

$$
u_{n}:=\widehat{\mu}\left(B\left(e, n^{-1}\right)\right)^{-1} \chi_{B\left(e, n^{-1}\right)},
$$


for all $\varrho_{0} \in \widehat{G}$ we have

$$
\begin{aligned}
\left(u_{n} * M\right)\left(\varrho_{0}\right) & =\int_{\widehat{G}} u_{n}\left(\varrho_{0} \varrho^{-1}\right) M(\varrho) d \widehat{\mu}(\varrho) \\
& =\widehat{\mu}\left(B\left(\varrho_{0}, n^{-1}\right)\right)^{-1} \int_{B\left(\varrho_{0}, n^{-1}\right)} M(\varrho) d \widehat{\mu}(\varrho) \\
& \stackrel{n}{\rightarrow} M\left(\varrho_{0}\right) \quad \text { if } \varrho_{0} \text { is a Lebesgue point of } M .
\end{aligned}
$$

Now we write $u_{n}=\mathcal{F}\left(\phi_{n}\right) \psi_{n}$, where

$$
\phi_{n}:=\widehat{\mu}\left(B\left(e, n^{-1}\right)\right)^{-1} \mathcal{F}^{-1}\left(\chi_{B\left(e, n^{-1}\right)}\right) \quad \text { and } \quad \psi_{n}:=\chi_{B\left(e, n^{-1}\right)} .
$$

For all Lebesgue points $\varrho_{0}$ of $M$ and all $x \in X$ we obtain

$$
\begin{aligned}
M\left(\varrho_{0}\right) x & \stackrel{n}{\leftarrow}\left(M * u_{n}\right)\left(\varrho_{0}\right) x \\
& =\int_{\widehat{G}} M(\varrho) \tau_{\varrho_{0}} u_{n}(\varrho) d \widehat{\mu}(\varrho) x \\
& =\int_{\widehat{G}} M(\varrho) \tau_{\varrho_{0}} \mathcal{F}\left(\phi_{n}\right)(\varrho) \tau_{\varrho_{0}} \psi_{n}(\varrho) d \widehat{\mu}(\varrho) x \\
& =\int_{\widehat{G}} M(\varrho) \mathcal{F}\left(\varrho_{0} \phi_{n} \otimes x\right)(\varrho) \tau_{\varrho_{0}} \psi_{n}(\varrho) d \widehat{\mu}(\varrho) \\
& =\int_{G} T_{M}\left(\varrho_{0} \phi_{n} \otimes x\right)(g) \overline{\mathcal{F}^{-1}\left(\tau_{\varrho_{0}} \psi_{n}\right)(g)} d \mu(g) \\
& =\int_{G}\left(\varrho_{0} T_{M} \varrho_{0}\right)\left(\phi_{n} \otimes x\right)(g) \overline{\mathcal{F}^{-1}\left(\psi_{n}\right)(g)} d \mu(g) .
\end{aligned}
$$

Here $\tau_{\varrho_{0}}$ denotes the translation operator $\tau_{\varrho_{0}} f(\varrho):=f\left(\varrho \varrho_{0}^{-1}\right)$. Since $T_{M} \in$ $\mathfrak{L}\left(L_{p}(G ; X)\right)$ by hypothesis, from Remark $3.3(\mathrm{f})$ we deduce that

$$
\sigma:=\left\{g T_{M} h:\|g\|_{L_{\infty}(G)},\|h\|_{L_{\infty}(G)} \leq 1\right\} \text { is R-bounded }
$$

in $\mathfrak{L}\left(L_{p}(G ; X)\right)$. Thus for all $N \in \mathbb{N}, x_{1}, \ldots, x_{N} \in X$ and Lebesgue points $\varrho_{1}, \ldots, \varrho_{N}$ of $M$ we get

$$
\begin{aligned}
& \int_{0}^{1}\left\|\sum_{j=1}^{N} r_{j}(t) M\left(\varrho_{j}\right) x_{j}\right\|^{p} d t \\
& \quad=\lim _{n} \int_{0}^{1}\left\|\int_{G} \sum_{j=1}^{N} r_{j}(t)\left(\varrho_{j} T_{M} \varrho_{j}\right)\left(\phi_{n} \otimes x_{j}\right)(g) \overline{\mathcal{F}^{-1}\left(\psi_{n}\right)(g)} d \mu(g)\right\|^{p} d t \\
& \quad \leq \sup _{n} \int_{0}^{1} \int_{G}\left\|\sum_{j=1}^{N} r_{j}(t)\left(\varrho_{j} T_{M} \varrho_{j}\right)\left(\phi_{n} \otimes x_{j}\right)(g)\right\|^{p} d \mu(g) d t\left\|\mathcal{F}^{-1}\left(\psi_{n}\right)\right\|_{p^{\prime}}^{p}
\end{aligned}
$$




$$
\begin{aligned}
& \leq R_{p}(\sigma)^{p} \sup _{n} \int_{0}^{1} \int_{G}\left\|\sum_{j=1}^{N} r_{j}(t) \phi_{n}(g) x_{j}\right\|^{p} d \mu(g) d t\left\|\mathcal{F}^{-1}\left(\psi_{n}\right)\right\|_{p^{\prime}}^{p} \\
& =R_{p}(\sigma)^{p} \int_{0}^{1}\left\|\sum_{j=1}^{N} r_{j}(t) x_{j}\right\|^{p} d t \sup _{n}\left\|\phi_{n}\right\|_{p}^{p}\left\|\mathcal{F}^{-1}\left(\psi_{n}\right)\right\|_{p^{\prime}}^{p}
\end{aligned}
$$

The proof is finished in view of our hypothesis $\sup _{n}\left\|\phi_{n}\right\|_{p}\left\|\mathcal{F}^{-1}\left(\psi_{n}\right)\right\|_{p^{\prime}}$ $<\infty$.

REMARK 5.1. If $p \in(1, \infty)$ and $G \in\left\{\mathbb{R}^{N}, \mathbb{T}^{N}, \mathbb{Z}^{N}\right\}$ for some $N \in \mathbb{N}$, then hypothesis (7) of Proposition 1.4 is satisfied, i.e.

$$
\sup _{n \in \mathbb{N}} \widehat{\mu}\left(B_{\widehat{G}}\left(e, n^{-1}\right)\right)^{-1}\left\|\mathcal{F}^{-1}\left(\chi_{B_{\widehat{G}}\left(e, n^{-1}\right)}\right)\right\|_{L_{p}(G)}\left\|\mathcal{F}^{-1}\left(\chi_{B_{\widehat{G}}\left(e, n^{-1}\right)}\right)\right\|_{L_{p^{\prime}}(G)}<\infty .
$$

Proof. Let $f_{n}:=\mathcal{F}^{-1}\left(\chi_{B_{\widehat{G}}}\left(e, n^{-1}\right)\right)$. For the case $G=\mathbb{T}^{N}$ we observe that $B_{\mathbb{Z}^{d}}\left(0, n^{-1}\right)=\{0\}$ and thus $f_{n}(z) \equiv C_{N}$ for all $n \geq 2$ and $z \in G$.

For the case $G=\mathbb{R}^{N}$ note that $f_{n}(\xi)=n^{-N} f_{1}\left(n^{-1} \xi\right)$ by dilation, hence $\left\|f_{n}\right\|_{q}=n^{-N(1-1 / q)}\left\|f_{1}\right\|_{q}$, and the assertion follows from

$$
\left|B_{\mathbb{R}^{N}}\left(0, n^{-1}\right)\right|^{-1}=C_{N}^{\prime} n^{N} \quad \text { for all } n \in \mathbb{N} .
$$

For the case $G=\mathbb{Z}^{N}$ we consider the metric $\widehat{d}$ on $\mathbb{T}^{N}$ defined by

$$
\widehat{d}(e, \exp (i x)):=\|x\|_{\infty}, \quad x \in(-\pi, \pi]^{N},
$$

and the requirement of translation invariance. Then for all $k \in \mathbb{Z}^{N}$ we have

$$
\begin{aligned}
f_{n}(k) & =C_{N}^{\prime \prime} \int_{[-\pi, \pi]^{N}} \exp (-i k x) \chi_{\left[-n^{-1}, n^{-1}\right]^{N}}(x) d x \\
& =C_{N}^{\prime \prime} \prod_{j=1}^{N} \int_{-n^{-1}}^{n^{-1}} \exp \left(-i k_{j} t\right) d t=2 C_{N}^{\prime \prime} \prod_{j=1}^{N} k_{j}^{-1} \sin \left(k_{j} n^{-1}\right) .
\end{aligned}
$$

Thus the assertion follows as above from the following estimate, where $\preceq$ means domination up to constants depending only on $N$ and $q$ :

$$
\begin{aligned}
\left\|f_{n}\right\|_{q} & \preceq\left(\sum_{k \in \mathbb{N}^{N}} \prod_{j=1}^{N} k_{j}^{-q}\left|\sin \left(k_{j} n^{-1}\right)\right|^{q}\right)^{1 / q} \\
& =\left(\sum_{m \in \mathbb{N}} m^{-q}\left|\sin \left(m n^{-1}\right)\right|^{q}\right)^{N / q} \\
& \leq\left(\sum_{m=1}^{n} n^{-q}+\sum_{m=n+1}^{\infty} m^{-q}\right)^{N / q} \preceq n^{-N(1-1 / q)} .
\end{aligned}
$$

6. Proof of Theorem 1.1. By hypothesis, $T \in \mathfrak{L}(X)$ is power-bounded and analytic, hence $\left(e^{t(T-I)}\right)$ is a bounded analytic semigroup by Nevan- 
linna's Theorem 2.3. Therefore, $(\mathrm{d}) \Leftrightarrow(\mathrm{e}) \Leftrightarrow(\mathrm{f})$ follow directly from Weis' Corollary 4.4 in [W1], cited as Theorem A in our Introduction.

(a) $\Leftrightarrow(b)$. By definition, $T$ has discrete maximal regularity if and only if the "convolution operator" on $\mathbb{Z}_{+}$,

$$
\begin{gathered}
f \mapsto\left(\sum_{n=0}^{m} k_{T}(n) f_{m-n}\right)_{m \in \mathbb{Z}_{+}}, \\
k_{T}: \mathbb{Z} \rightarrow \mathfrak{L}(X), \quad n \mapsto \begin{cases}(T-I) T^{n} & \text { for } n \in \mathbb{N}_{0}, \\
0 & \text { otherwise },\end{cases}
\end{gathered}
$$

is bounded on $l_{p}\left(\mathbb{Z}_{+} ; X\right)$ for some $p \in(1, \infty)$. By Remark 2.2 and

$$
\widehat{k}_{T}(z)=z((z-1) R(z, T)-I), \quad z \in \mathbb{T},
$$

the latter can be expressed equivalently in terms of Fourier multipliers:

$$
T_{M} \in \mathfrak{L}\left(l_{p}(X)\right), \quad \text { where } \quad \mathcal{F}\left(T_{M} f\right)(z):=(z-1) R(z, T) \widehat{f}(z) .
$$

But, by Proposition 1.4, (18) implies that

$$
\{(z-1) R(z, T): z \in \mathbb{T}, z \neq 1\} \text { is R-bounded, }
$$

hence $(\mathrm{a}) \Rightarrow(\mathrm{b})$ is already established. Conversely, (19) implies that the sets $\{M(t): t \in(0,2 \pi)\}$ and $\left\{\left(e^{i \theta}-1\right) M^{\prime}(t): t \in(0,2 \pi)\right\}$ are R-bounded for $M(t):=\left(e^{i \theta}-1\right) R\left(e^{i \theta}, T\right)$ and that (18) holds by the Mikhlin Theorem 1.3. Therefore, also $(\mathrm{b}) \Rightarrow(\mathrm{a})$ is established.

(c) $\Rightarrow(\mathrm{f})$. Let $\tau:=\left\{T^{n},(n+1)(T-I) T^{n}: n \in \mathbb{N}_{0}\right\}$ be R-bounded. Since $e^{t(T-I)}=e^{-t} \sum_{n=0}^{\infty} \frac{t^{n}}{n !} T^{n}, \quad t(T-I) e^{t(T-I)}=e^{-t} \sum_{n=0}^{\infty} \frac{t^{n+1}}{(n+1) !}(n+1)(T-I) T^{n}$, the operators $e^{t(T-I)}$ and $t(T-I) e^{t(T-I)}$ for $t>0$ belong to the closure of the absolute convex hull of $\tau$, which is R-bounded by Remark 3.3(b).

$(\mathrm{e}) \Rightarrow(\mathrm{b})$. Let the set $\{(\lambda-1) R(\lambda, T): \lambda \in 1+i \mathbb{R}, \lambda \neq 1\}$ be R-bounded. Then $\left\{(\lambda-1) R(\lambda, T): \lambda \in 1+\Sigma_{\delta}\right\}$ is R-bounded for some $\delta>0$ by the "maximum principle" Remark 3.3(d) and the "sector extension" Corollary 3.5(a). Since $\{R(\lambda, T):|\lambda|=1,|\arg (\lambda)| \geq \varepsilon\}$ is R-bounded for all $\varepsilon>0$ by Corollary 3.5(b), we obtain the R-boundedness of $\{(\lambda-1) R(\lambda, T):|\lambda|=1, \lambda \neq 1\}$.

(b) $\Rightarrow$ (c) is shown in Proposition 3.7 .

Acknowledgements. The author was supported by a scholarship of the "Gemeinsames Hochschulsonderprogramm III von Bund und Ländern" by the German Academic Exchange Office (DAAD). The author is deeply indebted to Prof. T. Coulhon for proposing him the problem of discrete maximal regularity and for the support during his postdoctoral fellowship at the University of Cergy-Pontoise. 


\section{References}

[B] S. Blunck, Analyticity and discrete maximal regularity on $L_{p}$-spaces, J. Funct. Anal., to appear.

[BCP $]$ A. Benedek, A. P. Calderón and R. Panzone, Convolution operators on Banach space valued functions, Proc. Nat. Acad. Sci. U.S.A. 48 (1962), 356-365.

[BG] E. Berkson and T. A. Gillespie, Spectral decompositions and harmonic analysis on UMD spaces, Studia Math. 112 (1994), 13-49.

[Bou] J. Bourgain, Vector-valued singular integrals and the $H^{1}-B M O$ duality, in: Probability Theory and Harmonic Analysis, Monogr. Textbooks Pure Appl. Math. 98, Dekker, 1986, 1-19.

[CP] P. Clément and J. Prüss, An operator-valued transference principle and maximal regularity on vector-valued $L_{p}$-spaces, preprint, 1999.

[C] T. Coulhon, Random walks and geometry on infinite graphs, in: Proc. Spring School "Analysis on Metric Spaces", Trento, 1999.

[CG] T. Coulhon and A. Grigoryan, Random walks on graphs with regular volume growth, Geom. Funct. Anal. 8 (1998), 656-701.

[CL] T. Coulhon et D. Lamberton, Régularité $L^{p}$ pour les équations d'évolution, Séminaire d'Analyse Fonctionnelle 1984/1985, Publ. Math. Univ. Paris VII 26, Univ. Paris VII, 1986, 155-165.

[C-SC] T. Coulhon et L. Saloff-Coste, Puissances d'un opérateur régularisant, Ann. Inst. H. Poincaré Probab. Statist. 26 (1990), 419-436.

[CV] P. Cannarsa and V. Vespri, On maximal $L^{p}$ regularity for the abstract Cauchy problem, Boll. Un. Mat. Ital. B (6) 5 (1986), 165-175.

[D] G. Dore, $L^{p}$ regularity for abstract differential equations, in: Functional Analysis and Related Topics, H. Komatsu (ed.), Lecture Notes in Math. 1540, Springer, 1993, 25-38.

[EG] R. E. Edwards and G. I. Gaudry, Littlewood-Paley and Multiplier Theory, Springer, 1977.

[H-SC] W. Hebisch and L. Saloff-Coste, Gaussian estimates for Markov chains and random walks on graphs, Ann. Probab. 21 (1993), 673-709.

[KL] N. Kalton and G. Lancien, A solution to the problem of maximal $L^{p}$-regularity, Math. Z. 235 (2000), 559-568.

[KT] Y. Katznelson and L. Tzafriri, On power bounded operators, J. Funct. Anal. 68 (1986), 313-328.

[L] D. Lamberton, Equations d'évolution linéaires associées à des semi-groupes de contractions dans les espaces $L^{p}$, ibid. 72 (1987), 252-262.

[LT] J. Lindenstrauss and L. Tzafriri, Classical Banach Spaces II (Function Spaces), Springer, 1979.

[Ly] Yu. Lyubich, Spectral localization, power-boundedness and invariant subspaces under Ritt's type condition, Studia Math. 134 (1999), 153-167.

[N1] O. Nevanlinna, Convergence of Iterations for Linear Equations, Birkäuser, Basel, 1993.

[N2] - On the growth of the resolvent operators for power bounded operators, in: Linear Operators, J. Janas, F. H. Szafraniec and J. Zemánek (eds.), Banach Center Publ. 38, Inst. Math., Polish Acad. Sci., 1997, 247-264.

[NZ] B. Nagy and J. Zemánek, A resolvent condition implying power boundedness, Studia Math. 134 (1999), 143-151.

[P] A. Pazy, Semigroups of Linear Operators and Applications to Partial Differential Equations, Springer, 1983. 
[SW] Z. Strkalj and L. Weis, On operator-valued Fourier multiplier theorems, preprint, 1999.

[W1] L. Weis, Operator-valued Fourier multiplier theorems and maximal $L^{p}$-regularity, Math. Ann., to appear.

[W2] -, A new approach to maximal regularity, in: Proc. 6th Internat. Conf. on Evolution Equations and their Applications in Physical and Life Sciences (Bad Herrenalb, 1998), G. Lumer and L. Weis (eds.), Marcel Dekker, 2000, to appear.

Département des Mathématiques

Université de Cergy-Pontoise

2, avenue Adolphe Chauvin

95302 Cergy-Pontoise, France

E-mail: Soenke.Blunck@math.u-cergy.fr

Received July 31, 2000

Revised version January 2, 2001 